\title{
Designing, Implementing and Testing a Mobile Application to Assist with Pediatric-to-Adult Health Care Transition
}

\author{
Jeremy Dixon ${ }^{1}$, Josh Dehlinger ${ }^{1}$, and Shannan DeLany Dixon ${ }^{2}$ \\ ${ }^{1}$ Department of Computer and Information Sciences, Towson University, Towson, MD, USA \\ jdixon6estudents.towson.edu, \\ jdehlinger@towson.edu \\ ${ }^{2}$ Department of Pediatrics, University of Maryland - School of Medicine, Baltimore, MD, USA \\ smdixonasom. umaryland. edu
}

\begin{abstract}
As development of mobile applications continues to expand, accessibility and utility for users who are differently-abled will become essential. One aspect that impacts a large portion of the differently-abled population is the process of medical transition. Medical transition for patients with chronic diseases from pediatric-based care to adult-based care is one that has been studied, developed and implemented for a number of years; recently, it has become a top priority in healthcare. Due to the complexities of the transition process, a well-designed, intuitive mobile application may improve the standardization and ease of care for these patients. This paper proposes and analyzes the design for a mobile transition navigator application (MTNA) while taking into account some of the most common considerations when working with differently-abled users. Specifically, three aspects of mobile application design are examined: (1) mobile user interfaces are different than traditional interfaces; 2. a variety of mobile platforms exist; and, 3. mobile platforms generate benefits and concerns such as the wide variety of screen sizes and resolutions.
\end{abstract}

Keywords: mobile applications, differently-abled technologies, humancomputer interaction, accessibility.

\section{Introduction}

As mobile applications become ubiquitous in our everyday lives, accessibility and utility for users who are differently-abled becomes essential [1]. Both Google and Apple have spent considerable resources including new accessibility tools into their most recent versions of their mobile operating systems in order to help support the differently-abled populations [2][3]. One important topic that impacts a large portion of the differently-abled population is the process of medical transition. The period of transition is when an individual is required to move from one healthcare team to another. In the medical field, transitions commonly occur when a young adult transitions from pediatric care to adult care, typically between the ages of 18 and 21 
[4]. If the transition fails or is incomplete, it may cause physical and/or mental health issues for adolescents with chronic conditions [5]. Medical transition for patients with chronic diseases from pediatric-based care to adult-based care is one that has been studied, developed and implemented for a number of years; recently, it has become a top priority in healthcare [4]. Due to the complexities of the transition process, a welldesigned, intuitive mobile application should improve the standardization and ease of care for these patients. The significance of this applied research is to bring needed attention to the evaluation and necessity of a mobile application that assists with pediatric-to-adult transition which is considered to be a critical issue in healthcare today.

Helping children and young adults with chronic conditions (e.g., people with inborn errors of metabolism, such as phenylketonuria or individuals who have Down syndrome) transition from their pediatric care team to an adult care team is a much more common occurrence than it was just thirty years ago [6]. For children without known chronic medical issues (including physical, emotional, or behavioral issues), transition to adult medical care is typically not a task that is noteworthy or challenging. Specifically, most typically functioning individuals would be expected to identify and meet with a new primary care physician, typically an internal medicine specialist, without much thought or planning [6].

A mobile application is being developed and will be tested on a small pilot study group after the application has been implemented. This paper provides a design framework for an application to be used on a tablet computer to help with the medical transition for young adults with a chronic disease. The work presented in this paper is a part of a larger effort to enable a smooth transition for children with chronic diseases to adult care through a comprehensive, usable mobile application.

The remainder of the paper is organized as follows. Section II provides the background of the pediatric-to-adult care transition process. Section III discusses the Mobile Transition Navigator Application (MTNA) and the related software engineering considerations. Section IV introduces additional considerations such as mobile user interfaces, the different mobile platforms, and hardware differences. Section V examines the pilot study and the expected outcomes. Section VI provides some concluding remarks and thoughts. Finally, the resources appear in Section VII.

\section{Transition}

The primary period of transition in medicine occurs when an adolescent transfers from pediatric to adult care between the ages of 18 and 21 [4]. A transition that provides uninterrupted, high-quality care is an important goal, yet it can be inconsistent. According to the American Academy of Pediatrics, "The goal of a planned health care transition is to maximize lifelong functioning and well-being for all youth, including those who have special health care needs and those who do not" [4]. For people with chronic medical conditions, this transition process is both complex and critically important [6] [7]. Transition most commonly involves the following key topics: 
1. Transferring care between large health care teams which may include multiple physicians, allied health professionals, nurses, and counselors [4].

2. Changing insurance coverage and practices.

3. Learning to discuss the disease, medications, and self-promotion.

4. Helping to ensure that all aspects of the transition are completed via checklists.

Orchestrating the transition from a pediatric team to an adult care team requires significant planning and execution. In order to help minimize obstacles and maximize independence required to successfully implement and monitor this transition, a mobile application will be designed for users who may be differently-abled.

As the number of children with chronic medical conditions who are surviving into adulthood increases, it is becoming essential to create and maintain an efficient transition process that is patient driven. Adolescents with a wide variety of chronic conditions make up almost one-fifth of children (18\%) in the United States [8]. Transition plans are often complex because they need to integrate patients, caregivers, and health care professionals. As the child transitions to adult care, health care providers who frequently have a different philosophy and approach to medicine than their pediatric counterparts often assume responsibility for the now adult patient [8]. These adult medicine health care providers may not be familiar with the nuances of the patient's particular condition as well as current treatment and management regimens. This can make the transition challenging for both the patient and the medical care team.

Many chronic conditions require a team of health care providers to meet the unique needs of all of the prospective issues that may arise. The loss of a pediatrician and pediatric subspecialists can be a traumatic experience for the adolescent because the pediatric provider is likely to have had a long-standing relationship with the patient and family. In some cases, specialists (or subspecialists) may act as the primary care physician due to the complexities of the condition which may complicate the coordination of the transition. Also, finding an adult facility that has all of the required specialties and subspecialties may be difficult, especially in more remote areas [9].

A failed transition process can have severe effects on the physical and mental health of adolescents with chronic conditions. Many adolescents with a chronic health care condition have established a long-term relationship with their pediatrician and other health care providers [5]. Research suggests that most pediatricians and internal medicine doctors do not feel comfortable providing primary care for young adults with chronic illnesses of childhood origin. For example, only $15 \%$ of internists stated that they would feel comfortable providing primary care for a patient with cystic fibrosis compared to $38 \%$ of pediatricians. Similarly, $32 \%$ of internists thought they would feel comfortable providing primary care to an adult with sickle cell disease compared to $35 \%$ of pediatricians [10].

As many parents become comfortable with their child's health care providers over time, it becomes more difficult to try and motivate individuals to transfer care from their current pediatric care team for a variety of reasons. First, people are resistant to change especially when it involves their children who have chronic medical 
conditions. Second, the transition process is complex as it involves changing doctors, locations of care sites, reeducation, and changes to insurance coverage. Transition is sometimes initiated by the change in insurance that occurs when the patient reaches the ages of 18-21, which is when many children are no longer eligible to receive coverage under a parental policy [9]. This motivation for transition may be changed by the Patient Protection and Affordable Care Act that was signed into law on March 23, 2010; this law increases the dependent age limit to 26 [11]. Lastly, patients may not feel pressured to complete transition by the pediatric team and so lack motivation to undergo the complex transition process.

\section{Mobile Transition Navigator Application}

The proposed mobile application will be called the Mobile Transition Navigator Application (MTNA). It is being designed to help children and young adults facilitate the transition process. Children and young adults with chronic conditions may also have a wide variety of visual, hearing and motor impairments. Thorough evaluation of the accessibility of the MTNA during the design process will improve ease of use for the intended users. Similar accessibility considerations that affect web design may also affect mobile application design because of the user diversity.

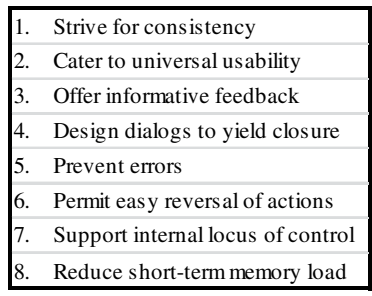

Fig. 1. Schneiderman's Eight Golden Rules [12]

As technology within tablet computing advances, a primary challenge is to identify novel research within the human-computer interaction subspecialty. Additionally, as this young field continues to evolve, additional resources will be identified and assessed for interface design and utility. A significant piece of interface design research, not specific to tablet computing, has been reported by Shneiderman and colleagues at the University of Maryland. The research identified Eight Golden Rules of Interface Design (Figure 1) [12] that one must adhere to for a well-designed interface. These rules should be considered during the design of the MTNA.

As discussed in Schneiderman's Eight Golden Rules, designing a consistent display can increase the usability of the application [12]. There are several aspects relating to the organization of the display that need to be taken into consideration. As the MTNA will be required to gather data from the users based on responses to the transition plan, it is important that the display is well organized. Smith and Mosier identified several aspects of data display to be taken into consideration. Most importantly, data displayed needs to be consistent. That is, each aspect of the interface should use the same vocabulary, abbreviations, form design, colors, and navigation [13]. 
Designing an appropriate user interface that is usable has additional complications as the size of tablet devices vary in size from $\sim 5 "$ to $\sim 10$ ". Importantly, because the user interface will be accessed using a multi-touch interface, the menu system needs to take into consideration the size of the user's forefinger as well as gross and fine motor skills. Research by Chen et al. suggests that for users without disability, a button size of 0.78 " is optimal while users with a disability's performance continued to improve as button size increased [14]. Specifically, the menu system should provide an icon or click point to on the interface which is easily accessible by people with a variety of finger sizes and mobility. Tablet devices have the ability to be used in either a portrait or a landscape orientation. There is a significant difference whether the screen is 9.5 " $\mathrm{x} 7.5$ " or 7.5" x 9.5" regarding the amount of text that is available on the screen. By taking advantage of some of the larger screens greater than 10", some of the accessibility considerations may be improved as the screen will be larger.

The MTNA will use a 2D menu structure using a broad hierarchy. This will allow the full height of the screen to be maximized so that scrolling will be reduced. The primary, and most commonly used parts of the tool, will be emphasized [15].

Data entry will be done by either tapping a virtual keyboard or by using Swype technology [16]. Swype technology allows users to drag their fingers across a virtual keyboard rather than having to lift their fingers up between characters. As an add-on to the virtual keyboard that is common on mobile devices, Swype does not interfere with more traditional tapping [16].

From an accessibility standpoint, children and young adults with chronic conditions may have a wide variety of visual, hearing and motor impairments. Because so many prospective users may have accessibility requirements, evaluating accessibility considerations during the design improves the available population of testers. Some of the same accessibility considerations that affect web design may also affect mobile application design because of the user diversity. In this section, some of the more common accessibility considerations are discussed for a mobile application.

The web content accessibility guidelines (WCAG 2.0) describe a variety of recommendations for making web content more accessible [17]. While these are intended for use on a web site, many of the same accessibility concerns may affect mobile application. For applications that use any non-text content (e.g., images, graphs, or figures), it is important to provide a means with which to translate that content into a form more usable by users. Users with visual impairment may require the text or text alternatives to be in large print or Braille. Other users may require textto-speech or symbols. Creating a clearly distinguished foreground and background can also help make the screen more easily viewed. It is important to choose a color scheme that is easily readable for users who may have partial or full color blindness. For visually impaired users, providing a mechanism for resizing the text will be worthwhile consideration. Some users who have chronic conditions may have an increased risk of seizures. Thus, the applications designed for this population should not have content that can increase the likelihood of seizures. Specifically, applications should not flash more than three times in a one second period [17]. Additionally, a small study conducted by the University of Washington suggests that screen readers, voice input, large buttons, screen magnification, improved contrast, and optical 
character recognition may considered for designing an application for differentlyabled [18].

The MTNA will aid with transition by helping the patient answer some of the critical key questions. Based on recommendations from the American Academy of Pediatrics, the application will help guide a patient through four main components of transition. First, the application will illustrate key questions to help decide if a patient is ready to initiate the transition process. If the patient is not ready to start the transition process, the application will provide guidance as to what tasks need to be established prior to starting the transition. Second, the application will help a patient plan the transition process. It will provide suggestions for goals along with realistic timelines. The main goal of this phase is to help educate a patient as to what is involved in a successful transition. Third, the application will help initiate patient transition. The primary goal of the implementation phase is to educate all involved parties as to the situation. In many cases, this will require the patient, caregivers, family members and the healthcare team to be educated as to a patient's condition, the expectations of care, and the timeline for completion. The application will provide a list of resources, and talking points to help with the implementation phase but will not provide any disease-specific resources. Finally, the application will help document important aspects of the transition process, such as important insurance information, contact information for the healthcare team, and checklists to help ensure that nothing is missed [4].

\section{Additional Considerations}

The widespread availability of mobile devices introduced a number of challenges to traditional software engineering, including: 1 . mobile user interfaces are different than traditional interfaces; 2 . a variety of mobile platforms exist; and, 3. mobile platforms generate benefits and concerns such as the variety of screen sizes and resolutions [1].

There are a wide variety of mobile devices that could be used with many differently-abled users. By using a mobile device, it is expected that users will increase their efficiency by having increased access to the computing device [19]. Further, many mobile devices have a variety of features to help the differently-abled interact with a mobile device. Specifically, many mobile devices have the ability to use text-to-speech, haptic feedback, alternative input devices (trackballs and D-pads) and audio feedback.

Most table devices today have a multi-touch interface that would be compatible with a wide variety of application designs. In order to meet the needs of all of the transitional aspects of the MTNA, the devices will have to have at least a 9" multitouch screen with WiFi. In order to keep the maintenance and services costs of the pilot study at a minimum, as well as maximizing connectivity, the devices will not be required to be $3 \mathrm{G}$ or $4 \mathrm{G}$ cell enabled. Based on current advances in the mobile device chip technology, tablet speed is not expected to be a consideration for this application.

One of the initial considerations for developing for mobile devices is that there are two distinct options: native and web [20]. Native applications are programs that use the programming language most commonly associated with the operating system 
(OS) of the device. For these native applications, the device supports embedded software development kits (SDK). The most prominent are Android SDK, iOS SDK and BlackBerry Java SDK. For the most part, native applications are different for each of the operating systems. Specifically, an application developed for the Android OS has to be recoded for a device running iOS. Native applications are often installed directly onto the mobile application by way of an App Store (i.e., Google Play or iTunes). A mobile web application, however, is designed to run from a web browser. Web based applications can be installed directly on the device or accessed from a remote server. In many cases, these web applications can run across devices and operating systems with minimal additional code but without the ability to use some of the native rich functionality.

Recently, software reusability options for mobile application development have improved because of technologies such as PhoneGap and Sencha Touch [21]. In the early days of iOS, Android, and Blackberry, application development was difficult because each application needs to be developed for each platform. PhoneGap, Sencha and other similar cross-compilation technologies allow the developer to code an application once that is then usable across platforms.

Sencha Touch uses a Model-View-Controller (MVC) architecture that is a design pattern that separates data from an application, the user interface, and the logic of business in three components. The origin of the MVC architecture or paradigm goes back to 1979 but the concepts introduced then are valid in modern mobile device software development. The three primary objects to the MVC each provide a specialized task. The visual representation (view) provides feedback to the user is the first task. The view would display the images and textual output. The second task connects the user input from a mouse, a keyboard, or a touchscreen to the model and the view [22]. Of the third task, the model, Burbeck states, "Finally, the model manages the behavior and data of the application domain, responds to requests for information about its state (usually from the view), and responds to instructions to change state (usually from the controller)" [22].

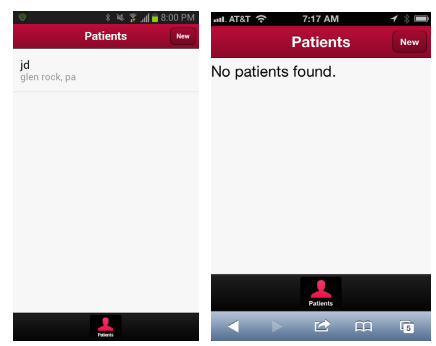

Fig. 2. Screenshots of the MTNA on both Android (left) and iOS (right)

Figure 2 shows the prototype MTNA on an Android-based phone and an Applebased phone as developed using Sencha Touch 2. The application can run on all HTML 5 enabled phones, tablets and personal computers.

Ultimately, Sencha Touch allows developers to create HTML5 based mobile apps that work on Android, iOS and Blackberry devices. While the application was not 
developed specifically for the device specific OS, in some cases it can take advantage of native functionality. Since Sencha Touch can be used to create an application that is cross-platform compatible while leveraging the MVC architecture, it will be used to create the MTNA application.

\section{$5 \quad$ Pilot Study}

The application will be tested using a small cohort of young adult patients, between the ages of 14-25 years old. While the users are new to the MNTA application, they are expected to have some familiarity with touch screens as most individuals in this demographic have access to or experiences with this technology. Successful implementation of the MTNA will be determined by the following criteria:

- Users will successfully complete their transition in less than 6 months.

- Users will have no lapses in medical care during the transition process and for the six months after the transition has been completed.

- The MTNA will receive generally positive reviews from both the patients and care givers.

The data collected from the pilot study will be analyzed using a mixed methodology. 1. survey data will evaluate the ease and success of the transition process when using the MTNA; and, 2. semi-structured interviews will collect comments, suggestions, and future features for the MTNA and will undergo thematic analysis. This combination of data will help drive future direction of the MTNA.

The survey will generate data assessing overall ease of usability and frequency of application use for the participants. Additionally, it will assess perceived helpfulness of the application and motivation to use the application based on physical access to a tablet computer. Participants will be asked to report the frequency of use of the transition timelines and self-assessment check lists as well as degree of helpfulness of discussion question prompts. Lastly, participants will be asked to propose additions to the application as well as features that should be considered for elimination from the application.

While survey data is collected, participants will be asked to participate in semi structured interviews. For those that agree, interviews will be used to explore and expand themes identified in the survey as well as allow for participants to further qualify feedback regarding the application so as not to be limited to only those choices provided in survey responses. The interview structure will also allow for participants to propose additional concepts that should be considered in development of the application. Lastly, the interviews will provide feedback regarding the benefits and limitations of medical transition using a phone or tablet based application.

In addition to the pilot study, data will be collected during a more traditional usability study. The usability study will be used on a variety of adolescents both typically functioning and differently abled. Tasks and/or interviews evaluating efficiency, accuracy, recall, and emotional response will be conducted prior to the pilot study. Additional data such as demographics will be included as part of the usability study. 


\section{Conclusion}

The best transition experiences are those where the care is uninterrupted and high quality. The design, implementation and testing of a mobile application may significantly help with the transition process. By leveraging modern crosscompilation tools, the MTNA application can be implemented on multiple mobile operating systems. This will lead to a variety of users, including those who may be differently-abled, to have a more fluid transition to their new health care providers.

Providing high quality healthcare to a person going through the transition from pediatric care to adult care can be a complex and time consuming effort. The best transition experiences are those where the care is uninterrupted and high quality. Currently, there are a variety of checklists and tools available to help people who are going through the transition period to complete the process but with varying results.

Advances in medical knowledge and skills are helping children and young adults with chronic conditions live much longer than in previous generations. Due to this, the population of people going through the transition process is increasing. By designing a mobile application framework that helps with the transition process, the efforts required to successfully complete this may be improved. Once the application is developed and validated, the application may be distributed to a broader patient base throughout the medical system to determine effectiveness to a global patient population.

Acknowledgements. We would like to acknowledge New York Mid Atlantic Consortium for Genetics and Newborn Screening Services (NYMAC) for their support and funding of this project.

\section{References}

1. Dehlinger, J., Dixon, J.: Mobile Application Software Engineering: Challenges and Research Directions. In: MobiCase 2011 - Mobile Software Engineering Workshop, Santa Monica (2011)

2. Apple Inc. iOS Accessibility (2013), https: / / developer.apple.com/technologies/ios / accessibility.html

3. Google Inc. Android 4.2, Jelly Bean - Accessibility (2013), http: / /www . android.com/about/jelly-bean/

4. American Academic of Pediatrics, Supporting the Transition from Adolescence to Adulthood in the Medical Home. Pediatrics, 182-200 (2011)

5. Binks, J.A., Barden, W.S., Burke, T.A., Young, N.L.: What Do We Really Know About the Transition to Adult-Centered Health Care? A Focus on Cerebral Palsy and Spina Bifida. Archives of Physical Medicine Rehabilitation 88, 1064-1073 (2007)

6. Peter, N., Forke, C., Ginsburg, K., Schwarz, D.: Transition From Pediatric to Adult Care: Internists' Perspectives. Pediatrics, 417-423 (2009)

7. Lotstein, D.S., McPherson, M., Strickland, B., Newacheck, P.W.: Transition Planning for Youth With Special Health Care Needs: Results from the National Survey of Children With Special Health Care Needs. Pediatrics 115(6), 1562-1568 (2005) 
8. Callahan, S.T., Feinstein Winitzer, R., Kennan, P.: Transition from Pediatric to Adult Oriented Health Care: A Challenge for Patients with Chronic Disease. Current Opinions in Pediatrics 13, 310-316 (2001)

9. American Academy of Pediatrics, Transition of Care Provided for Adolescents with Special Health Care Needs. Pediatrics, 1203-1206 (1996)

10. Okumura, M., et al.: Comfort of General Internists and General Pediatricians in Providing Care for Young Adults with Chronic Illnesses of Childhood. Journal of General Internal Medicine 23(10), 1621-1627 (2008)

11. U.S. Department of Health \& Human Services. Young Adults and the Affordable Care Act (August 2011), http: / / www . healthcare.gov/news / factsheets / 2011/08/young-adults.html

12. Shneiderman, B., Plaisant, C.: Designing the User Interface, 5th edn. Addison Wesley, Boston (2010)

13. Smith, S., Mosier, J.: Guidelines for Designing User Interface Software, Bedford, Massachusetts, USA (1986)

14. Chen, K., Savage, A., Chourasia, A., Wiegmann, D., Sesto, M.: Touch Screen Performance by Individuals With and Without Motor Control Disabilities. Applied Ergonomics 44, 297-302 (2013)

15. Kim, K., Jacko, J., Salvendy, G.: Menu Design for Computers and Cell Phones: Review and Reappriasal. International Journal of Human-Computer Interaction, 383-404 (2011)

16. King, I.: Spreading: Swype's Touchscreen Keyboard Technology, Businessweek.com (2010)

17. Caldwell, B., Cooper, M., Reid, L.G., Vanderheiden, G.: Web Content Accessibility Guidelines (WCAG) 2.0 (December 2008), http: / / www . w3 . org / TR/WCAG2 0 /

18. Kane, S., Jayant, S., Wobbrock, J., Ladner, R.: Freedom to roam: a study of mobile device adoption and accessibility for people with visual and motor disabilities. ACM, Pittsburgh (2009)

19. Martins, H., Jones, M.: Relevance of Mobile Computing in the Field of Medicine. In: Mobile Computing: Concepts, Methodologies, Tools, and Application, pp. 1429-1441 (2009)

20. Sutter, B.: HTML5 for the Java Web Developer (2012),

http: / / vimeo.com/29403374

21. Christ, A.: Bridging the Mobile App Gap, pp. 27-32. Inside the Digial Ecosystem, Sigma (2011)

22. Burbeck, S.: Applications Programming in Smalltalk-80: How to use Model-ViewController (MVC) (1987),

http://st-www.cs.illinois.edu/users/smarch/st-docs/mvc.html 